\title{
Log-Sobolev Inequalities for Infinite One Dimensional Lattice Systems
}

\author{
Boguslaw Zegarlinski ${ }^{\star}$ \\ Institute of Mathematics, Ruhr-Universität, D-4630 Bochum, FRG \\ Received September 19, 1989; in revised form February 14, 1990
}

Abstract. It is shown that a unique Gibbs measure of infinite spin system with short range interaction on one dimensional lattice satisfies log-Sobolev inequality.

\section{Introduction}

Log-Sobolev inequalities (shortly log-S) have been introduced in [1] and since then many investigations of them and related problems have been published. (For a recent bibliographical review see [2].) Although one of the important features of these inequalities is the fact that they generalize the classical Sobolev inequalities to infinite dimensional spaces, there are only few papers dealing specifically with the infinite dimensional case. Let us shortly describe them. In [1] it has been shown that any infinite product of probability measures $\left\{\rho_{n}\right\}_{n \in \mathbf{N}}$ satisfies log-S inequality with a coefficient $0<c<\infty$, provided each measure $\rho_{n}$ satisfies $\log -\mathrm{S}$ with a corresponding coefficient $0<c_{n}<c$.

Moreover, using this fact, in the same paper it has been proven that also any Gaussian measure satisfies log-S. This, together with a general theory developed in [1], yields an elegant proof of hypercontractivity estimates of Nelson [3] (see also [4]) for the free field, so important in development of euclidean field theory.

The first example of probability measures on an infinite dimensional space satisfying log-Sobolev inequalities and not being of product or Gaussian type appeared in [5]. The authors used the $\Gamma_{2}$-criterion of Bakry and Emery [6] to prove these inequalities for the measures of classical statistical mechanical systems on a lattice with single spin space given by the $S^{d}$-sphere $d \geqq 2$ and at sufficiently high temperatures. The authors of this paper, Carlen and Stroock, were motivated by investigation of Markov semigroups and in particular by applications to the study of stochastic dynamics in statistical mechanical systems ([7-12]).

\footnotetext{
* Supported by SFB 237
} 
More recently we have developed in $[13,14]$ another method for the investigation of log-Sobolev inequalities for probability measures on infinite dimensional spaces. Our method is based on the use of Gibbs structure $([15,16,17])$ together with a kind of Dobrushin uniqueness condition $[17,18,19]$. Using our method one can show log-S also in the cases when the $\Gamma_{2}$-criterion does not work (e.g. in [14] we deduced log-S inequalities for statistical mechanical systems on the lattice $\mathbf{Z}^{d}$ with single spin space $\{-1,+1\}$ ). One can apply this method together with a lattice approximation to show also that the probability measure of euclidean (quantum) field theory with $\lambda: \exp \alpha \phi:_{2}$ satisfies $\log -\mathrm{S}$ inequality with coefficient $m_{0}^{-2}$, with $m_{0}$ being a bare mass used to construct the probability measure in question. (Incidentally, one can also obtain this result by using the $\Gamma_{2}$-criterion.)

In the present paper we extend the ideas and methods of $[13,14]$ to show that each (infinite volume) Gibbs measure of one dimensional spin system with finite range interaction satisfies $\log -\mathrm{S}$ inequality. By this we settle also a problem raised in [12].

In order to formulate precisely our results we need to introduce some definitions and notations: We consider the integer lattice $\mathbf{Z}$. By $\mathscr{F}$ we denote the family of all finite subsets in $\mathbf{Z}$. Let $\mathscr{F}_{0}$ be an increasing sequence of intervals whose union contains all the lattice. We take a single spin space $\{-1,+1\}$ with discrete topology to define a space $\Omega \equiv\{-1,+1\}^{\mathbf{Z}}$ of configurations $\sigma$ of the infinite spin system. Let $\Sigma$ be the $\sigma$-algebra of subsets in $\Omega$ generated by the product topology. Let $\sigma_{i}: \Omega \rightarrow\{-1,+1\}, i \in \mathbf{Z}$, be the $i^{\text {th }}$ coordinate function, called a spin at site $i$. For $\Lambda \subset \mathbf{Z}$ we will use $\Sigma_{\Lambda}$ to denote $\sigma$-algebra of subsets of $\Omega$ generated by the functions $\left\{\sigma_{i}: i \in \Lambda\right\}$.

If a function $f$ is $\Sigma_{\Lambda}-$ (respectively $\Sigma-$ ) measurable we write $f \in \Sigma_{\Lambda}$ (respectively $f \in \Sigma$ ). By $\mu_{0}$ we will denote the free measure on $(\Omega, \Sigma)$ defined as the product of uniform probability measures on $\{-1,+1\}$. For any probability measure $\mu$ on $(\Omega, \Sigma)$, the expectation value of a function $f \in \Sigma$ with respect to $\mu$ is denoted by $\mu f$ or $\mu(f)$. A two point truncated correlation function of $f, g \in \Sigma$ is by definition

$$
\mu(f, g) \equiv \mu f g-\mu f \mu g .
$$

For further purposes we define a "differentiation" $B_{i}$ with respect to the $i^{\text {th }}$ coordinate as the projector on nonconstant functions with respect to $\sigma_{i}$ given by

$$
B_{i} f \equiv \frac{1}{2}\left(f_{\mid \sigma_{i}=+1}-f_{\mid \sigma_{i}=-1}\right) \sigma_{i},
$$

where $f_{\mid \sigma_{i}=+1}$ denotes the evaluation of the function $f$ on $\Omega$ at the point $\sigma \in \Omega$ with $\sigma_{i}=+1$, and similarly for $f_{\mid \sigma_{i}=-1}$. We set also

$$
A_{i} \equiv 1-B_{i}
$$

and define for $\Lambda \subset \mathbf{Z}$

$$
\left|B_{\Lambda} f\right|^{2} \equiv \sum_{i \in \Lambda}\left|B_{i} f\right|^{2}
$$

If $\Lambda=\mathbf{Z}$ we will write simply $B \equiv B_{\mathbf{Z}}$.

Let $\mathscr{M}$ denote the set of bounded measurable real functions on $(\Omega, \Sigma)$. By definition an interaction is a function

$$
\Phi: \mathscr{F} \rightarrow \mathscr{M}
$$


An interaction $\Phi$ has finite range $r \equiv r(\Phi)$ iff there exist $r \in \mathbf{N}$ which is a smallest number such that

$$
\Phi_{X} \equiv 0
$$

for $X \in \mathscr{F}, \operatorname{diam}(X) \geqq r$, where $\operatorname{diam}(X)$ is the diameter of the set $X$. $\Phi$ is called translation invariant iff

$$
\Phi_{X+a}(\sigma .)=\Phi_{X}(\sigma \cdot-a)
$$

for any $X \in \mathscr{F}$ and $a \in \mathbf{Z}$.

We will assume that the norm of interaction we consider, defined by

$$
\|\Phi\| \equiv \sup _{i \in \mathbf{Z}} \sum_{\substack{X \in \mathscr{F} \\ i \in X}}\left\|\Phi_{X}\right\|_{\infty},
$$

is finite ( \|\|$_{\infty}$ denotes the supremum norm). This is of course satisfied for finite range translation invariant interactions. Since the case $r=1$ is trivial (then we have a product measure already discussed in [1]) we restrict ourselves to the interactions with range $r>1$. For an interaction $\Phi$ we define an interaction functional $U_{\Lambda}$ at a volume $\Lambda \in \mathscr{F}$ by

$$
U_{\Lambda} \equiv \sum_{X \cap \Lambda \neq \varnothing} \Phi_{X}
$$

Now let us introduce the probability kernels

$$
E_{\Lambda}^{\sigma}(\cdot):=\delta_{\sigma} \frac{\mu_{0_{\mid \Lambda}}\left(e^{-U_{\Lambda}}\right)}{\mu_{0_{\mid \Lambda}}\left(e^{-U_{\Lambda}}\right)},
$$

where $\Lambda \in \mathscr{F}, \delta_{\sigma}$ is the point measure concentrated at $\sigma \in \Omega$ and $\mu_{0_{\mid \Lambda}}$ denotes the conditional expectation of $\mu_{0}$ with respect to $\Sigma_{\Lambda^{c}}$.

The family $\mathscr{E} \equiv\left\{E_{\Lambda}^{\cdot}\right\}_{\Lambda \in \mathscr{F}}$ forms a local specification in the sense of $[15,16]$, i.e. it satisfies:

i) for any $f \in \Sigma$ and $\Lambda \in \mathscr{F}$

$$
E_{\Lambda}^{\cdot} f \in \Sigma_{\Lambda^{c}}
$$

and if $f \in \Sigma_{\Lambda^{c}}$ then

$$
E_{\Lambda}^{\sigma} f=f(\sigma)
$$

ii) (Compatibility condition) for any $\Lambda, \Lambda^{\prime} \in \mathscr{F}, \Lambda \subset \Lambda^{\prime}$,

$$
E_{\Lambda^{\prime}}^{\sigma} E_{\Lambda}^{*}=E_{\Lambda^{\prime}}^{\sigma}
$$

A probability measure $\mu$ on $(\Omega, \Sigma)$ satisfying

$$
\mu E_{\Lambda}=\mu
$$

for all $\Lambda \in \mathscr{F}$ is called $a$ Gibbs measure for $\mathscr{E}$. The set of Gibbs measures for a local specification $\mathscr{E}$ is denoted by $\mathscr{G}(\mathscr{E})$.

It is known (see e.g. [20]) that in one dimension for a finite range interaction $\mathscr{G}(\mathscr{E})$ consists of a unique Gibbs measure $\mu$. Moreover this measure has an exponential decay of correlations, i.e.

$$
|\mu(f, g)| \leqq C\|f\|_{\infty}\|g\|_{\infty} e^{-m d(f, g)}
$$


with some constants $0<C, m<\infty$ independent of $f, g \in \Sigma$ and with

$$
d(f, g) \equiv \operatorname{dist} \quad(\operatorname{supp} f, \operatorname{supp} g) .
$$

A probability measure $\mu$ on $(\Omega, \Sigma)$ is said to satisfy a log-Sobolev inequality iff there is a constant $0<c<\infty$ such that

$$
\mu f^{2} \log |f| \leqq c \mu|B f|^{2}+\mu f^{2} \log \left(\mu f^{2}\right)^{1 / 2}
$$

for any function $f \in \Sigma$ for which $\mu|B f|^{2}<\infty$ and $\mu f^{2}<\infty$.

For investigation of Glauber's stochastic dynamics [21] associated with the spin system one uses a semigroup with generator $\mathscr{L}$ defined by

$$
\mathscr{L} f(\sigma):=\sum_{k \in \mathbf{Z}} c_{k}(\sigma)\left(f\left(\sigma^{k}\right)-f(\sigma)\right)
$$

for all functions $f \in \Sigma$ for which the right-hand side of $(0.16)$ is finite, where the functions $c_{k}(\sigma)$ are defined by

$$
c_{k} \equiv \frac{1}{2}\left(1-\frac{B_{k} e^{-U_{k}}}{A_{k} e^{-U_{k}}}\right)
$$

and

$$
\left(\sigma^{k}\right)_{i} \equiv \begin{cases}\sigma_{i} & \text { for } \quad i \neq k \\ -\sigma_{k} & \text { for } \quad i=k\end{cases}
$$

One can show, see e.g. $[7,8]$, that for any Gibbs measure $\mu$ one has

$$
\mu \mathscr{L} f=0
$$

and so any Gibbs measure is a stationary measure for the corresponding stochastic dynamics. We refer to [7-12] for an extensive study of stochastic dynamics. Let us note (see [7]) that for the quadratic form which is uniquely given by $-\mathscr{L}$ and the Gibbs measure $\mu$ one has

$$
\mu(f(-\mathscr{L} f))=\frac{1}{2} \sum_{k} \mu c_{k}(\sigma)\left(f\left(\sigma^{k}\right)-f(\sigma)\right)^{2}=2 \sum_{k} \mu c_{k}(\sigma)\left|B_{k} f(\sigma)\right|^{2} .
$$

Since by our assumption (0.7) and (0.17) we have

$$
0<\frac{1}{2}(1-t h\|\Phi\|) \leqslant c_{k}(\sigma) \leqslant 1,
$$

it follows that the inequality $(0.15)$ is equivalent with the following $\log -\mathrm{S}$ inequality for the Gibbs measure $\mu$

$$
\mu f^{2} \log |f| \leqslant c^{\prime} \mu(f(-\mathscr{L} f))+\mu f^{2} \log \left(\mu f^{2}\right)^{1 / 2},
$$

with the constant $0<c^{\prime}<\infty$ independent of function $f \in \Sigma$.

By the general theory developed in [1] the inequality (0.22) implies hypercontractivity of the semigroup $\exp (t \mathscr{L})$ and the existence of a mass gap for the generator $\mathscr{L}$ as an operator in $L^{2}(\mu)$. (By mass gap we understand as usual the gap in the spectrum of the positive self-adjoint operator $-\mathscr{L}$ in $L^{2}(\mu)$ between the infimum of its spectrum and the rest of the spectrum).

The main result of the present paper is summarized in the following theorem. 
Theorem 0.1. Let $\mu$ be a Gibbs measure of a one dimensional lattice spin system with finite range interaction of bounded norm. Then $\mu$ satisfies the log-Sobolev inequality.

Let us remark that due to the geometry of a one dimensional lattice no assumption on the temperature (as in $[13,14$ or 5$]$ ) is needed. We shall discuss the inequality $(0.15)$, but we should keep in mind that it is equivalent with $(0.22)$, which has direct application in statistical mechanics.

\section{Proof of Theorem 0.1}

The proof of our main result is based on some extension of ideas of [13] and [14] where was shown that $\log$-S satisfied by the finite volume kernels $E_{\Lambda}^{\sigma} \in \mathscr{E}$ with $|\Lambda|=n$ for some $n \in \mathbf{N}$, uniformly in boundary conditions $\sigma \in \Omega$, by some "generalized induction" imply log-S inequality for infinite volume measure $\mu \in \mathscr{G}(\mathscr{E})$. It was assumed there that the temperature of the spin system is sufficiently large. Here we take advantage of geometry of one dimension to obtain a stronger estimate sufficient to get the result for any temperature.

We consider an interaction $\Phi$ of finite range $r \equiv r(\Phi) \in \mathbf{Z}^{+}$satisfying

$$
\|\Phi\|<\infty
$$

with $\|\cdot\|$ given by (0.7). Let $L \in \mathbf{N}, L>1$. (This is a number we will control.) Let $\Gamma_{0} \equiv\left\{\Lambda_{k}\right\}_{k \in \mathbf{Z}}$ consist of intervals $\Lambda_{k} \equiv\left[a_{k}, b_{k}\right]$ where $a_{k}, b_{k} \in \mathbf{Z}, k \in \mathbf{Z}$ are restricted by the conditions

$$
\left.\begin{array}{rl}
b_{k}-a_{k} & =(2 L+1) r \\
a_{k+1}-b_{k} & =r \\
a_{1} & =r .
\end{array}\right\}
$$

We define also $\Gamma_{1} \equiv\left\{\tilde{\Lambda}_{k}\right\}_{k \in \mathbf{Z}}$ as a translation of $\Gamma_{0}$ by $(L+1) r$.

We note that the following fact holds:

Lemma 1.1. For any $\Lambda \in \Gamma_{i}(i=0,1)$ and any measurable function $f$ we have

$$
E_{\Lambda}^{\sigma} f^{2} \log |f| \leqq c_{0} E_{\Lambda}^{\sigma}\left|B_{\Lambda} f\right|^{2}+E_{\Lambda}^{\sigma} f^{2} \log \left(E_{\Lambda}^{\sigma} f^{2}\right)^{1 / 2},
$$

where

$$
0<c_{0} \leqq C \log |\Lambda|
$$

with a constant $0<C<\infty$ independent of $\Lambda, \sigma \in \Omega$ and a function $f$.

REM. As we shall see below it is sufficient for our purposes to have the estimate

$$
0<c_{0} \leqq \exp \left(C|\Lambda|^{\varepsilon}\right)
$$

with some $0<C<\infty$ and $0<\varepsilon<1$ independent of $\Lambda, \sigma \in \Omega$ and $f$.

Proof of Lemma 1.1. Under condition (1.1) on the finite range interaction, our lemma follows by use of property (0.21) and Lemma 1.5 in [12] (see also [11] Sect. 6) where the property (1.3) is shown for finite volume measures with the form of corresponding operator $-\mathscr{L}$ on right-hand side. 
Now let us define a sequence $\left\{\Gamma^{(k)}:=\Gamma_{\left(k \bmod _{2}\right)}\right\}_{k \in Z^{+}}$, where $k \bmod _{2}$ equals 0 for $k$ even and 1 otherwise. Similarly as in $[13,14]$ we have to consider the following arguments, based on the definition of the Gibbs measure (0.12) and use of the log-S inequality for conditional measures $E_{\Lambda}^{\sigma}, \Lambda \in \Gamma^{(k)}, k \in \mathbf{Z}^{+}$. For $\Lambda_{1} \in \Gamma^{(0)}$ we have

$$
\begin{aligned}
\mu f^{2} \log |f| & =\mu E_{\Lambda_{1}}^{\cdot} f^{2} \log |f| \leqq \mu\left[c_{0} E_{\Lambda_{1}}\left|B_{\Lambda_{1}} f\right|^{2}+E_{\Lambda_{1}} f^{2} \log \left(E_{\Lambda_{1}} f^{2}\right)^{1 / 2}\right] \\
& =c_{0} \mu\left|B_{\Lambda_{1}} f\right|^{2}+\mu\left(E_{\Lambda_{1}} f^{2} \log \left(E_{\Lambda_{1}} f^{2}\right)^{1 / 2}\right) .
\end{aligned}
$$

By applying the same argument to the second term on right-hand side (1.6) for some $\Lambda_{2} \in \Gamma^{(0)}$. We get

$$
\begin{aligned}
\mu f^{2} \log |f| \leqq & c_{0} \mu\left|B_{\Lambda_{1}} f\right|^{2}+c_{0} \mu\left|B_{\Lambda_{2}}\left(E_{\Lambda_{1}} f^{2}\right)^{1 / 2}\right|^{2} \\
& +\mu\left(E_{\Lambda_{2}} E_{\Lambda_{1}} f^{2} \log \left(E_{\Lambda_{2}} E_{\Lambda_{1}} f^{2}\right)^{1 / 2}\right) .
\end{aligned}
$$

Since $B_{\Lambda_{2}}$ affects only the function $f$ not the measures $E_{\Lambda_{1}}$, for $\Lambda_{1}, \Lambda_{2} \in \Gamma^{(0)}$, $\Lambda_{1} \neq \Lambda_{2}$, we have the estimate

$$
\left|B_{\Lambda_{2}}\left(E_{\Lambda_{1}} f^{2}\right)^{1 / 2}\right|^{2} \leqq E_{\Lambda_{1}}\left|B_{\Lambda_{2}} f\right|^{2} .
$$

This together with (1.7) gives

$$
\mu f^{2} \log |f| \leqq c_{0} \mu\left|B_{\Lambda_{1} \cup A_{2}} f\right|^{2}+\mu\left(E_{\Lambda_{2}} E_{\Lambda_{1}} f^{2} \log \left(E_{\Lambda_{2}} E_{\Lambda_{1}} f^{2}\right)^{1 / 2}\right) .
$$

An iteration of the above arguments leads to the inequality

$$
\mu f^{2} \log |f| \leqq c_{0} \mu\left|B_{\Gamma^{(0)}} f\right|^{2}+\mu\left(E_{\Gamma^{(0)}} f^{2} \log \left(E_{\Gamma^{(0)}} f^{2}\right)^{1 / 2}\right),
$$

where we used the notations

$$
\left|B_{\Gamma^{(0)}} f\right|^{2} \equiv \sum_{i \in \Gamma^{(0)}}\left|B_{i} f\right|^{2}
$$
(with an abuse of notation we used $\Gamma^{(0)}$ to denote $\left\{\bigcup_{k} \Lambda_{k}: \Lambda_{k} \in \Gamma^{(0)}\right\}$; we will keep
such a notation also in the rest of this paper) and

$$
E_{\Gamma^{(0)}} f^{2} \equiv \lim _{\substack{|n| \rightarrow \infty \\ \Lambda_{k} \in \Gamma^{(0)}, 0 \leqq|k| \leqq|n|}} E_{\Lambda_{n}} \cdots E_{\Lambda_{0}} f^{2}
$$

Note that the right-hand side of (1.12) is independent of the ordering of elements in $\Gamma^{(0)}$.

Now we take sets $\tilde{\Lambda}_{k} \in \Gamma^{(1)}$ and apply similar arguments to the second term on the right-hand side of (1.10). Afterwards we shall repeat all this with the sets from $\Gamma^{(2)}$ and iterate further the procedure. These inductive arguments after $N$ steps yield the following inequality

$$
\begin{aligned}
& \mu f^{2} \log |f| \leqq c_{0}\left\{\mu\left|B_{\Gamma^{(0)}} f\right|^{2}+\sum_{n=1}^{N} \mu\left|B_{\Gamma^{(n)}}\left(E_{\Gamma^{(n-1)}} \cdots E_{\Gamma^{(0)}} f^{2}\right)^{1 / 2}\right|^{2}\right\} \\
& +\mu\left(E_{\Gamma^{(N)}} \cdots E_{\Gamma^{(0)}} f^{2} \log \left(E_{\Gamma^{(N)}} \cdots E_{\Gamma^{(0)}} f^{2}\right)^{1 / 2}\right) .
\end{aligned}
$$

We will use the following lemma to control the right-hand side of (1.13) as $N \rightarrow \infty$.

\section{Lemma 1.2}

a) There is a constant $0<c_{1}<\infty$ such that

$$
\left|B_{\Gamma^{(1)}}\left(E_{\Gamma^{(0)}} f^{2}\right)^{1 / 2}\right|^{2} \leqq c_{1} E_{\Gamma^{(0)}}|B f|^{2}
$$

for any measurable function $f$. 
b) There is $L \in \mathbf{N}$ such that for any $f \in \sum_{\left(\Gamma^{(n)} \backslash \Gamma^{(n+1)}\right)}$

$$
\left|B_{\Gamma^{(n+1)}}\left(E_{\Gamma^{(n)}} f^{2}\right)^{1 / 2}\right|^{2} \leqq \lambda E_{\Gamma^{(n)}}\left|B_{\Gamma^{(n)}} f\right|^{2}
$$

with a constant $0<\lambda<1$ independent of $n \in \mathbf{Z}^{+}$and a function $f$.

We shall give the proof of Lemma 1.2 in a while, but first we like to show how using this lemma one completes the proof of Theorem 0.1 . For $n \geqq 2$ we set

$$
f_{n-2} \equiv\left(E_{\left.\Gamma^{(n-2)} \cdots E_{\Gamma^{(0)}} f^{2}\right)^{1 / 2}}\right. \text {. }
$$

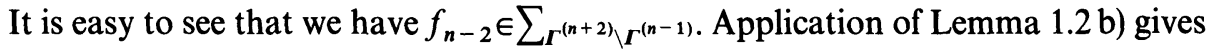

$$
\left|B_{\Gamma^{(n)}}\left(E_{\Gamma^{(n-1)}} f_{n-2}^{2}\right)^{1 / 2}\right|^{2} \leqq \lambda E_{\Gamma^{(n-1)}}\left|B_{\Gamma^{(n-1)}} f_{n-2}\right|^{2} .
$$

If $n>2$ we can repeat the arguments and by induction we get

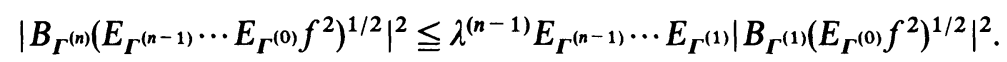

This together with Lemma 1.2 a) yields

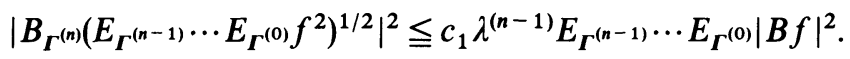

The inequality (1.19) has two consequences. First of all, as one can easily see, that its combination with property $(0.12)$ implies

$$
\lim _{n \rightarrow \infty} E_{\Gamma^{(n)}} \cdots E_{\Gamma^{(0)}} f^{2}=\mu f^{2}, \quad \mu \text {-a.e. }
$$

for any measurable $f$ such that $\mu|B f|^{2}<\infty$.

Secondly using (1.19) (and property $(0.12)$ ) we see that the sum in the curly bracket on the right-hand side of $(1.13)$ is bounded by

$$
\left[1+c_{1}(1-\lambda)^{-1}\right] \quad \mu|B f|^{2} .
$$

From (1.20), (1.21) and (1.13) we obtain the inequality

with

$$
\mu f^{2} \log |f| \leqq c \mu|B f|^{2}+\mu f^{2} \log \left(\mu f^{2}\right)^{1 / 2}
$$

$$
c \equiv c_{0}\left(1+c_{1}(1-\lambda)^{-1}\right)<\infty .
$$

This ends the proof of Theorem 0.1.

Proof of Lemma 1.2 a). Although we closely follow the arguments given in $[14,13]$, for the readers convenience, we present here a selfconsistent proof. In Lemma 1.3 below we show that for any $\Lambda_{k} \in \Gamma^{(0)}, \Lambda_{k} \equiv\left[a_{k}, b_{k}\right]$ and $j \in \Gamma^{(1)}, d\left(j, \Lambda_{k}\right) \leqq r$, the following inequality is true

$$
\left|B_{j}\left(E_{\lambda_{k}} f^{2}\right)^{1 / 2}\right| \leqq C_{1}\left(E_{\Lambda_{k}}\left|B_{j} f\right|^{2}\right)^{1 / 2}+C_{2}\left(E_{\Lambda_{k}}\left|B_{\Lambda_{k}} f\right|^{2}\right)^{1 / 2}
$$

with some constants $0<C_{1}, C_{2}<\infty$ independent of $\Lambda_{k}, j$ and any measurable function $f$. Suppose now that $j \in\left(b_{k}, a_{k+1}\right)$ (otherwise the left-hand side of (1.24) equals to zero). Let us set $\tilde{\Gamma} \equiv \Gamma^{(0)} \backslash\left(\Lambda_{k} \cup \Lambda_{k+1}\right)$. Using (1.24) we get

$$
\begin{aligned}
\left|B_{j}\left(E_{\Gamma^{(0)}} f^{2}\right)^{1 / 2}\right|= & \left|B_{j}\left(E_{\Lambda_{k}}\left(E_{\Lambda_{k+1}} E_{\tilde{\Gamma}} f^{2}\right)\right)^{1 / 2}\right| \\
\leqq & C_{1}\left(E_{\Lambda_{k}}\left|B_{j}\left(E_{\Lambda_{k+1}} E_{\tilde{\Gamma}} f^{2}\right)^{1 / 2}\right|^{2}\right)^{1 / 2} \\
& +C_{2}\left(E_{\Lambda_{k}}\left|B_{\Lambda_{k}}\left(E_{\Lambda_{k+1}} E_{\tilde{\Gamma}} f^{2}\right)^{1 / 2}\right|^{2}\right)^{1 / 2} .
\end{aligned}
$$


Taking into account that in the second term on the right-hand side of (1.25) $B_{A_{k}}$ affects only the function $f$ and not the measure $E_{\Lambda_{k+1}} E_{\tilde{\Gamma}}$, we can bound (1.25) as follows

$$
\left|B_{j}\left(E_{\Gamma^{(0)}} f^{2}\right)^{1 / 2}\right| \leqq C_{1}\left(E_{\lambda_{k}}\left|B_{j}\left(E_{\Lambda_{k+1}} E_{\tilde{\Gamma}} f^{2}\right)^{1 / 2}\right|^{2}\right)^{1 / 2}+C_{2}\left(E_{\Gamma^{(0)}\left|B_{\Lambda_{k}} f\right|^{2}}\right)^{1 / 2} .
$$

Applying (1.24) in the first term on the right-hand side of (1.26) and using the triangle inequality together with arguments similar to those used in passing from (1.25) to (1.26), we obtain

$$
\begin{aligned}
\left|B_{j}\left(E_{\Gamma^{(0)}} f^{2}\right)^{1 / 2}\right| \leqq & C_{1}^{2}\left(E_{\Gamma^{(0)} \mid}\left|B_{j} f\right|^{2}\right)^{1 / 2} \\
& +C_{1} C_{2}\left(E_{\Gamma^{(0)}}\left|B_{\Lambda_{k+1}} f\right|^{2}\right)^{1 / 2}+C_{2}\left(E_{\left.\Gamma^{(0)}\left|B_{\Lambda_{k}} f\right|^{2}\right)^{1 / 2}}\right.
\end{aligned}
$$

Squaring and summing over $j \in \Gamma^{(1)}$ we get the bound

$$
\begin{aligned}
& \left|B_{\Gamma^{(1)}}\left(E_{\Gamma^{(0)}} f^{2}\right)^{1 / 2}\right|^{2} \leqq 2 C_{1}^{4} E_{\Gamma^{(0)}}\left|B_{\Gamma^{(1)}} \Gamma^{(0)} f\right|^{2} \\
& +2 C_{2}^{2}\left(1+C_{1}^{2}\right) r 2^{(4 L+2) r} E_{\Gamma^{(0)} \mid}\left|B_{\Gamma^{(0)} f}\right|^{2} .
\end{aligned}
$$

Hence taking

$$
C_{1}=2 \max \left[C_{1}^{4}, C_{2}^{2}\left(1+C_{1}^{2}\right)^{r} 2^{(4 L+2) r}\right]
$$

we get Lemma 1.2a).

We shall now show that (1.24) is true.

Lemma 1.3. There are constants $0<C_{1}, C_{2}<\infty$ such that

$$
\left|B_{j}\left(E_{\Lambda_{k}} f^{2}\right)^{1 / 2}\right| \leqq C_{1}\left(E_{A_{k}}\left|B_{j} f\right|^{2}\right)^{1 / 2}+C_{2}\left(E_{A_{k}}\left|B_{A_{k}} f\right|^{2}\right)^{1 / 2}
$$

for any $\Lambda_{k}, j \in \boldsymbol{\Gamma}^{(1)}, d\left(j, \Lambda_{k}\right) \leqq r$ and any measurable function $f$.

Proof. Let us first observe that for any function $F$ we have

$$
B_{j} F^{2}=2 A_{j} F \cdot B_{j} F \text {. }
$$

Using this in order to get (1.30), it is sufficient to show that

$$
\left|B_{j} E_{\Lambda_{k}} f^{2}\right| \leqq 2 A_{j}\left(E_{\Lambda_{k}} f^{2}\right)^{1 / 2} \cdot[\operatorname{rhs}(1.30)] .
$$

By the definition of $B_{j}$ in $(0.2)$ we have

$$
B_{j}\left(E_{\Lambda_{k}} f^{2}\right) \equiv \frac{1}{2}\left[E_{\Lambda_{k} \mid \sigma_{j}=+1} f_{\mid \sigma_{j}=+1}^{2}-E_{\Lambda_{k} \mid \sigma_{j}=-1} f_{\mid \sigma_{j}=-1}^{2}\right] \cdot \sigma_{j} .
$$

We would like to study the right-hand side of (1.33) by using the fundamental theorem of calculus. To do that we introduce the interpolating functions

$$
f_{s_{j}}(\sigma) \equiv f\left(\sigma_{\mathbf{Z} \backslash}, s_{j}\right):=A_{j} f+\bar{B}_{j} f \cdot s_{j},
$$

where $s_{j} \in[-1,1]$ and

$$
\bar{B}_{j} f \equiv \frac{B_{j} f}{\sigma_{j}} .
$$

We need also interpolating measures $E_{\boldsymbol{A}_{k}, s_{j}}, s_{j} \in[-1,1]$ defined by setting in the definition (0.9)

$$
U_{\Lambda_{k}, s_{j}} \equiv U_{A_{k}}\left(\sigma_{\mathrm{Z} \backslash j}, s_{j}\right):=A_{j} U_{A_{k}}+\bar{B}_{j} U_{\Lambda_{k}} \cdot s_{j}
$$


Using this we have

$$
\left|B_{j} E_{\Lambda_{k}} f^{2}\right|=\left|\frac{1}{2} \int_{-1}^{1} d s_{j} 2 E_{\Lambda_{k}, s_{j}}\left(f_{s_{j}} \partial_{j} f_{s_{j}}\right)+\frac{1}{2} \int_{-1}^{1} d s_{j} E_{\Lambda_{k}, s_{j}}\left(f_{s_{j}}^{2}, \partial_{j} U_{\Lambda_{k}, s_{j}}\right)\right|
$$

with $\partial_{j} \equiv \frac{d}{d s_{j}}$.

Let us consider the first term on the right-hand side of (1.37). By Hoelder inequality we get

$$
\left|E_{\Lambda_{k}, s_{j}}\left(f_{s_{j}} \partial_{j} f_{s j}\right)\right| \leqq\left(E_{\Lambda_{k}, s_{j}} f_{s_{j}}^{2}\right)^{1 / 2}\left(E_{\Lambda_{k}, s_{j}}\left|\partial_{j} f_{s_{j}}\right|^{2}\right)^{1 / 2}
$$

Now observe that

$$
\partial_{j} f_{s_{j}}=\bar{B}_{j} f
$$

and that the density

$$
\rho_{\Lambda_{k}, s_{j}} \equiv \frac{d E_{\Lambda_{k}, s_{j}}}{d E_{\Lambda_{k}}}
$$

satisfies

$$
\max _{s_{j}, \sigma} \rho_{\Lambda_{k}, s_{j}} \leqq e^{4\|\Phi\|}
$$

From (1.38)-(1.41) we obtain

$$
\left|E_{\Lambda_{k}, s_{j}}\left(f_{s_{j}} \partial_{j} f_{s_{j}}\right)\right| \leqq e^{4\|\Phi\|}\left(E_{\Lambda_{k}} f_{s_{j}}^{2}\right)^{1 / 2}\left(E_{\Lambda_{k}}\left|B_{j} f\right|^{2}\right)^{1 / 2} .
$$

Let us now note that by Hoelder inequality we get

$$
\frac{1}{2} \int_{-1}^{1} d s\left(E_{\Lambda_{k}} f_{s_{j}}^{2}\right)^{1 / 2} \leqq\left(E_{\Lambda_{k}}\left(A_{j} f^{2}\right)\right)^{1 / 2}
$$

We have also

$$
\begin{aligned}
A_{j}\left(E_{\Lambda_{k}} f^{2}\right)^{1 / 2} & \equiv \frac{1}{2}\left[\left(E_{\Lambda_{k} \mid \sigma_{j}=+1} f_{\mid \sigma_{j}=+1}^{2}\right)^{1 / 2}+\left(E_{\Lambda_{k} \mid \sigma_{j}=-1} f_{\mid \sigma_{j}=-1}^{2}\right)^{1 / 2}\right] \\
& \geqq e^{-2\|\Phi\| \frac{1}{2}\left[\left(E_{\Lambda_{k}}\left(f_{\mid \sigma_{j}=+1}^{2}\right)\right)^{1 / 2}+\left(E_{\Lambda_{k}}\left(f_{\mid \sigma_{j}=-1}^{2}\right)\right)^{1 / 2}\right]} \\
& \geqq e^{-2\|\Phi\|}\left(E_{\Lambda_{k}}\left(\frac{1}{2} A_{j} f^{2}\right)\right)^{1 / 2} .
\end{aligned}
$$

Combining (1.38), (1.42)-(1.44) we obtain the bound

$$
\left|\frac{1}{2} \int_{-1}^{1} d s_{j} E_{\Lambda_{k}, s_{j}}\left(f_{s_{j}} \partial_{j} f_{s_{j}}\right)\right| \leqq A_{j}\left(E_{\Lambda_{k}} f^{2}\right)^{1 / 2} \cdot\left[2^{1 / 2} e^{6\|\Phi\|}\left(E_{\Lambda_{k}}\left|B_{j} f\right|^{2}\right)^{1 / 2}\right] .
$$

Now we shall consider the second term on the right-hand side of (1.37). We use the property (1.39) for $U_{\Lambda_{k}, s_{j}}$ and the identity

$$
E_{\Lambda_{k}, s_{j}}\left(f_{s_{j}}^{2}, \bar{B}_{j} U_{\Lambda_{k}}\right)=\frac{1}{2} E_{\Lambda_{k}, s_{j}} \otimes \tilde{E}_{\Lambda_{k}, s_{j}}\left(f_{s_{j}}^{2}(\sigma)-f_{s_{j}}^{2}(\tilde{\sigma})\right)\left(\bar{B}_{j} U_{\Lambda_{k}}(\sigma)-\bar{B}_{j} U_{\Lambda_{k}}(\tilde{\sigma})\right) \text {, }
$$

where $\sigma$ (respectively $\tilde{\sigma}$ ) is the integration variable with respect to $E_{\Lambda_{k}, s_{j}}$ (respectively $\widetilde{E}_{\Lambda_{k}, s_{j}}$ an isomorphic copy of $E_{\Lambda_{k}, s_{j}}$ ). To simplify the notation we will write $F$ 
respectively $\tilde{F}$ for $F(\sigma)$ respectively $F(\tilde{\sigma})$. From (1.46) one easily gets

$$
\begin{aligned}
\left|E_{\Lambda_{k}, s_{j}}\left(f_{s_{j}}^{2}, \bar{B}_{j} U_{\Lambda_{k}}\right)\right| & \leqq\|\Phi\| E_{\Lambda_{k}, s_{j}} \otimes \tilde{E}_{\Lambda_{k}, s_{j}}\left|f_{s_{j}}^{2}-\tilde{f}_{s_{j}}^{2}\right| \\
& \leqq 2\|\Phi\|\left(E_{\Lambda_{k}, s_{j}} f_{s_{j}}^{2}\right)^{1 / 2}\left(E_{\Lambda_{k}, s_{j}} \otimes \tilde{E}_{\Lambda_{k}, s_{j}}\left(f_{s_{j}}-\tilde{f}_{s_{j}}\right)^{2}\right)^{1 / 2}
\end{aligned}
$$

Now we use (1.41) to increase the right-hand side of (1.47) as follows:

$$
\left|E_{\Lambda_{k}, s_{j}}\left(f_{s_{j}}^{2}, \bar{B}_{j} U_{\Lambda_{k}}\right)\right| \leqq 2\|\Phi\| e^{6\|\Phi\|}\left(E_{\Lambda_{k}} f_{s_{j}}^{2}\right)^{1 / 2}\left(E_{\Lambda_{k}} \otimes \tilde{E}_{\Lambda_{k}}\left(f_{s_{j}}-\tilde{f}_{s_{j}}\right)^{2}\right)^{1 / 2}
$$

Integration of both sides of (1.48) with respect to $s_{j}$ and application of Hoelder inequality yield

$$
\begin{aligned}
&\left|\frac{1}{2} \int_{-1}^{1} d s_{j} E_{A_{k}, s_{j}}\left(f_{s_{j}}^{2}, B_{j} U_{\lambda_{k}}\right)\right| \leqq 2\|\Phi\| e^{6\|\Phi\|}\left(E_{\Lambda_{k}}\left(A_{j} f^{2}\right)\right)^{1 / 2} \\
& \cdot\left(E_{\Lambda_{k}} \otimes \tilde{E}_{\Lambda_{k}} A_{j}(f-\tilde{f})^{2}\right)^{1 / 2}
\end{aligned}
$$

From this, by similar arguments as in (1.44), we get

$$
\begin{aligned}
& \left|\frac{1}{2} \int_{-1}^{1} d s_{j} E_{\Lambda_{k}, s_{j}}\left(f_{s_{j}}^{2}, \partial_{j} U_{\Lambda_{k}, s_{j}}\right)\right| \\
& \quad \leqq 2 A_{j}\left(E_{\Lambda_{k}} f^{2}\right)^{1 / 2} \cdot\left[2^{1 / 2}\|\Phi\| e^{8\|\Phi\|}\left(E_{\Lambda_{k}} \otimes \widetilde{E}_{\Lambda_{k}} A_{j}(f-\tilde{f})^{2}\right)^{1 / 2}\right] .
\end{aligned}
$$

To estimate the last factor on the right-hand side of (1.50) let us note that

$$
\begin{aligned}
\left(E_{\Lambda_{k}} \otimes \tilde{E}_{\Lambda_{k}} A_{j}(f-\tilde{f})^{2}\right)^{1 / 2} & =\left(E_{\Lambda_{k}} \otimes \tilde{E}_{\Lambda_{k}}\left(\left(A_{j} f-A_{j} \tilde{f}\right)^{2}+\left(B_{j} f-B_{j} \tilde{f}\right)^{2}\right)\right)^{1 / 2} \\
& \leqq\left(E_{\Lambda_{k}} \otimes \tilde{E}_{\lambda_{k}}(f-\tilde{f})^{2}\right)^{1 / 2}+2\left(E_{\lambda_{k}} \otimes \tilde{E}_{\lambda_{k}}\left(B_{j} f-B_{j} \tilde{f}\right)^{2}\right)^{1 / 2} \\
& \leqq\left(E_{\Lambda_{k}} \otimes \tilde{E}_{\lambda_{k}}(f-\tilde{f})^{2}\right)^{1 / 2}+4\left(E_{\lambda_{k}}\left|B_{j} f\right|^{2}\right)^{1 / 2}
\end{aligned}
$$

Note also that since $E_{\Lambda_{k}}$ satisfies $\log$-S with coefficient $c_{0}$, so also $E_{\Lambda_{k}} \otimes \widetilde{E}_{\Lambda_{k}}$ satisfies that with the same coefficient. This, by arguments of [22] (see also [23]) implies the mass gap inequality

$$
2 E_{\Lambda_{k}} \otimes \tilde{E}_{\Lambda_{k}}(f-\tilde{f})^{2} \leqq c_{0} E_{\Lambda_{k}} \otimes \tilde{E}_{\Lambda_{k}}\left(\left|B_{\Lambda_{k}} f\right|^{2}+\left|B_{\Lambda_{k}} \tilde{f}\right|^{2}\right)=2 c_{0} E_{\Lambda_{k}}\left|B_{\Lambda_{k}} f\right|^{2}
$$

Therefore

$$
\left(E_{\Lambda_{k}} \otimes \tilde{E}_{\lambda_{k}}\left(A_{j}(f-\tilde{f})^{2}\right)\right)^{1 / 2} \leqq 4\left(E_{\lambda_{k}}\left|B_{j} f\right|^{2}\right)^{1 / 2}+c_{0}^{1 / 2}\left(E_{\lambda_{k}}\left|B_{\Lambda_{k}} f\right|^{2}\right)^{1 / 2}
$$

Inserting (1.53) into the right-hand side of (1.50) we obtain

$$
\begin{aligned}
& \left|\frac{1}{2} \int_{-1}^{1} d s_{j} E_{\Lambda_{k}, s_{j}}\left(f_{s_{j}}^{2}, \partial_{j} U_{\Lambda_{k}, s_{j}}\right)\right| \\
& \quad \leqq 2 A_{j}\left(E_{\Lambda_{k}} f^{2}\right)^{1 / 2} \cdot 2^{1 / 2}\|\Phi\| e^{8\|\Phi\|}\left[4\left(E_{\Lambda_{k}}\left|B_{j} f\right|^{2}\right)^{1 / 2}+c_{0}^{1 / 2}\left(E_{\Lambda_{k}}\left|B_{\Lambda_{k}} f\right|^{2}\right)^{1 / 2}\right] .
\end{aligned}
$$

This together with (1.45) and (1.37) yields the inequality (1.32), i.e.

with

$$
\left|B_{j} E_{\Lambda_{k}} f^{2}\right| \leqq 2 A_{j}\left(E_{\Lambda_{k}} f^{2}\right)^{1 / 2}\left\{C_{1}\left(E_{\Lambda_{k}}\left|B_{j} f\right|^{2}\right)^{1 / 2}+C_{2}\left(E_{\Lambda_{k}}\left|B_{\Lambda_{k}} f\right|^{2}\right)^{1 / 2}\right\}
$$

and

$$
C_{1} \equiv\left(4\|\Phi\| e^{2\|\Phi\|}+1\right) \cdot 2^{1 / 2} e^{6\|\Phi\|}
$$

$$
C_{2} \equiv\|\Phi\| e^{8\|\Phi\|} c_{0}^{1 / 2} \text {. }
$$

This ends the proof of Lemma 1.3 (and hence also of Lemma 1.2a). 
Proof of Lemma 1.2b. Let $f \in \sum_{\Gamma^{(n)} \backslash \Gamma^{(n+1)}}$. This means that

$$
\operatorname{supp} f \subset \Gamma^{(n)}
$$

and

$$
d\left(f, \mathbf{Z} \backslash \Gamma^{(n)}\right) \geqq L r .
$$

In this situation, for $j \in \Gamma^{(n)}$, we have

$$
B_{j} E_{\Gamma^{(n)}} f=0 .
$$

Let us consider $j \in \Gamma^{(n+1)} \backslash \Gamma^{(n)}$. Let us assume $n$ to be even and $j \in\left(b_{k}, a_{k+1}\right)$ for some $k \in \mathbf{Z}$ (the case $n$ odd can be treated similarly).

We follow the strategy of the proof of Lemma 1.3 and want to find a corresponding estimation of the form (1.32). Now we shall to consider the quantity

$$
\bar{B}_{j} E_{\Gamma^{(n)}} f^{2} \equiv \bar{B}_{j} E_{V_{k}} E_{\tilde{\Gamma}^{(n)}} f^{2}=\frac{1}{2}\left(E_{V_{k} \mid \sigma_{j}=+1} E_{\tilde{\Gamma}^{(n)}} f^{2}-E_{V_{k} \mid \sigma_{j}=-1} E_{\tilde{\Gamma}^{(n)}} f^{2}\right),
$$

where we have set

$$
V_{k} \equiv \Lambda_{k} \cup \Lambda_{k+1}
$$

and

$$
\tilde{\Gamma}^{(n)} \equiv \Gamma^{(n)} \backslash V_{k}
$$

Let us note that, due to the finite range of our interaction, we have

$$
\bar{B}_{j} E_{\Gamma^{(n)}} f^{2}=E_{\tilde{\Gamma}^{(n)}} \bar{B}_{j} E_{V_{k}} f^{2} .
$$

By using the fundamental theorem of calculus we obtain

$$
\bar{B}_{j} E_{V_{k}} f^{2}=\frac{1}{2} \int_{-1}^{1} d s_{j} E_{V_{k}, s_{j}}\left(f^{2}, \partial_{j} U_{V_{k}, s_{j}}\right)
$$

By a similar identity as (1.46) and application of (1.39) for $U_{V_{k}, s_{j}}$ we get

$$
E_{V_{k}, s_{j}}\left(f^{2}, \partial_{j} U_{V_{k}, s_{j}}\right)=\frac{1}{2} E_{V_{k}, s_{j}} \otimes \tilde{E}_{V_{k}, s_{j}}\left(f^{2}-\tilde{f}^{2}\right)\left(\bar{B}_{j} U_{V_{k}}-\bar{B}_{j} \tilde{U}_{V_{k}}\right) \text {. }
$$

From the definition of $U_{V_{k}}$ with a potential of range $r$ it follows that

$$
\operatorname{supp} \bar{B}_{j} U_{V_{k}} \subset\left(b_{k}-r, a_{k+1}+r\right)
$$

and so

$$
d\left(f, B_{j} U_{V_{k}}\right) \geqq(L-1) r,
$$

with $f$ here treated as a function of the integration variables only in $V_{k} \cap\left(\Gamma^{(n)} \backslash \Gamma^{(n+1)}\right)$. We would like to take advantage of (1.68) and the fact that our lattice is one dimensional to get a better bound on (1.66) than in the similar situation which occurred in the proof of Lemma 1.2a. To do that let us define the sets

$$
X_{l} \equiv\left[x_{l}, w_{l}\right] \cup\left[y_{l}, z_{l}\right] ; \quad l=1, \ldots, L+1
$$

by setting

$$
\left.\begin{array}{l}
x_{l} \equiv a_{k}+(L-1+l) r \\
w_{l} \equiv x_{l}+r
\end{array}\right\}
$$


and

$$
\left.\begin{array}{l}
y_{l} \equiv a_{k+1}+(L+2-l) r \\
z_{l} \equiv y_{l}-r
\end{array}\right\}
$$

We set also

$$
X_{L+2} \equiv\left(b_{k}, a_{k+1}\right)
$$

Let for $i=0, \ldots, L$,

$$
V_{k, i} \equiv V_{k} \backslash \bigcup_{i+1}^{L+1} X_{l}
$$

We need also to introduce the following notation: For $l=1, \ldots, L+1$,

and

$$
H_{l} \equiv \sum_{X \cong X_{l}} \Phi_{X}
$$

$$
W_{l, l+1} \equiv \sum_{\substack{X \cap X_{l} \neq \varnothing \\ X \cap X_{l+1} \neq \varnothing}} \Phi_{X}
$$

We will define the interpolating functions as in the proof of Lemma 1.3. With the notation just introduced we have

$$
U_{V_{k}, s_{j}}=U_{V_{k, 0}}+\sum_{l=1}^{L+1} H_{l}+\sum_{l=1}^{L} W_{l, l+1}+W_{L+1, L+2, s_{j}}
$$

Let us observe that by the definition of $E_{V_{k}}^{\bar{\sigma}}$ with a fixed external conditions $\bar{\sigma} \in \Omega$ we have

$$
\begin{aligned}
E_{V_{k}, s_{j}}^{\bar{\sigma}} \otimes \tilde{E}_{V_{k}, s_{j}}^{\bar{\sigma}}\left(f^{2}-\tilde{f}^{2}\right)\left(\bar{B}_{j} U_{V_{k}}-\bar{B}_{j} \tilde{U}_{V_{k}}\right) \\
=\frac{1}{\left(Z_{V_{k}, s_{j}}^{\tilde{\sigma}}\right)^{2}} \delta_{\tilde{\sigma}} \otimes \tilde{\delta}_{\tilde{\sigma}}\left\{\mu _ { 0 _ { | V _ { k } } } \otimes \tilde { \mu } _ { 0 _ { | V _ { k } } } \left(e^{-\left(U_{V_{k}, s_{j}}+\tilde{U}_{V_{k}, s_{j}}\right)}\left(f^{2}-\tilde{f}^{2}\right)\right.\right. \\
\left.\left.\quad \cdot\left(\bar{B}_{j} U_{V_{k}}-\bar{B}_{j} \tilde{U}_{V_{k}}\right)\right)\right\},
\end{aligned}
$$

where $\left(Z_{V_{k}, s_{j}}^{\bar{\sigma}}\right)^{-2}$ is a normalization factor. Let us compute the expectation with respect to the point measure $\delta_{\bar{\sigma}} \otimes \widetilde{\delta}_{\bar{\sigma}}$ on the right-hand side of (1.75) and consider $U_{V_{k}, s_{j}}$ and $U_{V_{k}}$ as functions on the configurations $\left\{\sigma \in \Psi: \sigma_{\mid V_{k}^{c}} \equiv \bar{\sigma}_{\mid V_{k}^{c}}\right\}$. For simplicity of notation, from now on we will suppress explicit dependence on $\bar{\sigma}_{\mid V_{k}^{c}}$. Let us consider the curly bracket on the right-hand side of (1.75) (with evaluated expectation with respect to the point measure). Using the fact that the interaction is of finite range $r$, by taking the conditional expectation associated to the measure $\mu_{0_{\mid V_{k}}} \otimes \tilde{\mu}_{0_{\mid V_{k}}}$ with respect to $\sigma$-algebra $\Sigma\left(\bigcup_{l \geqq 2} X_{l}\right)$, we can represent this curly bracket
as follows:

$$
\begin{aligned}
& \mu_{0_{\mid V_{k} \backslash V_{k, 1}}} \otimes \tilde{\mu}_{0_{\mid V_{k} \backslash V_{k, 1}}}\left(F^{(1)}(\sigma, \tilde{\sigma})\left(\bar{B}_{j} U_{V_{k}}-\bar{B}_{j} \tilde{U}_{V_{k}}\right)\right. \\
& \left.\quad \cdot \exp \left(-\left(U_{s_{j}}\left(V_{k} \backslash V_{k, 2}\right)+\tilde{U}_{s_{j}}\left(V_{k} \backslash V_{k, 2}\right)+H_{2}+\tilde{H}_{2}\right)\right)\right),
\end{aligned}
$$

where

$$
F^{(1)}(\sigma, \tilde{\sigma}) \equiv \delta_{\sigma} \otimes \tilde{\delta}_{\tilde{\sigma}}\left(\mu_{0_{\mid V_{k, 1}}} \otimes \tilde{\mu}_{0_{\mid V_{k, 1}}}\left(f^{2}-\tilde{f}^{2}\right) e^{-\left(U\left(V_{k, 1}\right)+\tilde{U}\left(V_{k, 1}\right)\right)}\right)
$$


and where we used, for typographical reasons, the notation $U_{s_{i}}\left(V_{k} \backslash V_{k, 1}\right)$ respectively $U\left(V_{k, 1}\right)$ as subscripts and similarly with $V_{k, 2}$. The functions $F^{(1)}(\sigma, \tilde{\sigma})$ have two important properties. First of all, due to the finite range of interaction, we have

$$
F^{(1)}(\sigma, \tilde{\sigma})=F^{(1)}\left(\sigma_{X_{2}}, \tilde{\sigma}_{X_{2}}\right)
$$

i.e. $F^{(1)}$ is a function only of the spins in the set $X_{2}$ (if $\bar{\sigma}$ is fixed). Moreover $F^{(1)}$ satisfies

$$
F^{(1)}\left(\sigma_{X_{2}}, \tilde{\sigma}_{X_{2}}\right)=-F^{(1)}\left(\tilde{\sigma}_{X_{2}}, \sigma_{X_{2}}\right)
$$

Therefore we can write it in the form

$F^{(1)}\left(\sigma_{X_{2}}, \tilde{\sigma}_{X_{2}}\right)=\mu_{0_{\mid V_{k, 1}}} \otimes \tilde{\mu}_{0_{\mid V_{k, 1}}}\left(\left(f^{2}-\tilde{f}^{2}\right) G_{1,2}^{-} \exp \left(-\left(U\left(V_{k, 0}\right)+\tilde{U}\left(V_{k, 0}\right)+H_{1}+\tilde{H}_{1}\right)\right)\right)$,

where $G_{1,2}^{-}$is a particular case of the following notation:

$$
\begin{aligned}
G_{l, l+1}^{ \pm} \equiv & \frac{1}{2}\left[\exp \left(-W_{l, l+1}\left(\sigma_{X_{l}}, \sigma_{X_{l+1}}\right)-W_{l, l+1}\left(\tilde{\sigma}_{X_{l}}, \tilde{\sigma}_{X_{l+1}}\right)\right)\right. \\
& \left. \pm \exp \left(-W_{l, l+1}\left(\sigma_{X_{l}}, \tilde{\sigma}_{X_{l+1}}\right)-W_{l, l+1}\left(\tilde{\sigma}_{X_{l}}, \sigma_{X_{l+1}}\right)\right)\right]
\end{aligned}
$$

Now we consider (1.76) and compute the conditional expectation of the integrand with respect to $\Sigma\left(\bigcup_{l \geqq 3} X_{l}\right)$. This gives

$$
\begin{aligned}
& (1.76)=\mu_{0_{\mid V_{k} \backslash V_{k, 2}}} \otimes \tilde{\mu}_{0_{V_{k} \backslash V_{k, 2}}}\left(F^{(2)}(\sigma, \tilde{\sigma})\left(\bar{B}_{j} U_{V_{k}}-\bar{B}_{j} \tilde{U}_{V_{k}}\right)\right. \\
& \left.\cdot \exp \left(-\left(U_{s_{j}}\left(V_{k} \backslash V_{k, 3}\right)+\tilde{U}_{s_{j}}\left(V_{k} \backslash V_{k, 3}\right)+H_{3}+\tilde{H}_{3}\right)\right)\right) \text {, }
\end{aligned}
$$

with

$$
F^{(2)}(\sigma, \tilde{\sigma})=F^{(2)}\left(\sigma_{X_{3}}, \tilde{\sigma}_{X_{3}}\right) \equiv \mu_{0_{\mid X_{2}}} \otimes \tilde{\mu}_{0_{\mid X_{2}}}\left(F^{(1)}\left(\sigma_{X_{2}}, \tilde{\sigma}_{X_{2}}\right) G_{2,3}^{-} \cdot e^{-\left(H_{X_{2}}+\tilde{H}_{X_{2}}\right)}\right) .
$$

Applying inductively these arguments we see that the expectation in the curly bracket on the right-hand side of (1.75) equals

$$
\begin{aligned}
& \mu_{0_{\mid V_{k}}} \otimes \tilde{\mu}_{0_{\mid V_{k}}}\left(\left(f^{2}-\tilde{f}^{2}\right) \prod_{l=1}^{L} G_{l, l+1}^{-}\left(\bar{B}_{j} V_{k}-\bar{B}_{j} \tilde{U}_{V_{k}}\right)\right. \\
& \left.\quad \exp \left(-\left(\sum_{l=1}^{L+1}\left(H_{l}+\tilde{H}_{l}\right)+U\left(V_{k, 0}\right)+\tilde{U}\left(V_{k, 0}\right)+W_{L+1, L+2, s_{j}}+\tilde{W}_{L+1, L+2, s_{j}}\right)\right)\right) .
\end{aligned}
$$

We bound the absolute value of (1.84) by taking the absolute value of the integrand in this expectation. Multiplying and dividing the integrand of the estimator obtained in this way by the quanity

$$
\prod_{l=1}^{L} G_{l, l+1}^{+}
$$

we obtain

$$
\left(Z_{V_{k}, s_{j}}^{\bar{\sigma}}\right)^{-2} \cdot|(1.84)| \leqq E_{V_{k}, s_{j}} \otimes \tilde{E}_{V_{k}, s_{j}}\left(\left|f^{2}-\tilde{f}^{2}\right| \prod_{l=1}^{L} \gamma_{l}\left|\bar{B}_{j} U_{V_{k}}-\bar{B}_{j} \tilde{U}_{V_{k}}\right|\right)
$$


with

$$
\gamma_{l} \equiv\left|\frac{G_{l, l+1}^{-}}{G_{l, l+1}^{+}}\right| .
$$

From this, (1.75)-(1.84) we get

$$
|(1.75)| \leqq\left\|\bar{B}_{j} U_{V_{k}}-\bar{B}_{j} \tilde{U}_{V_{k}}\right\|_{\infty} \cdot \gamma^{L} E_{V_{k}, s_{j}} \otimes \tilde{E}_{V_{k}, s_{j}}\left|f^{2}-\tilde{f}^{2}\right|
$$

with

$$
\gamma \equiv \max _{l}\left\|\gamma_{l}\right\|_{\infty}
$$

Let us note that by the very definition of $\gamma$ we have

$$
0<\gamma<1 \text {. }
$$

Now we take into account that

$$
E_{V_{k}, s_{j}} \otimes \widetilde{E}_{V_{k}, s_{j}}\left|f^{2}-\tilde{f}^{2}\right| \leqq e^{4\|\Phi\|} E_{V_{k}} \otimes \tilde{E}_{V_{k}}\left|f^{2}-\tilde{f}^{2}\right|
$$

and

$$
\begin{aligned}
E_{V_{k}} \otimes \tilde{E}_{V_{k}}\left|f^{2}-\tilde{f}^{2}\right| & \leqq\left(E_{V_{k}} \otimes \tilde{E}_{V_{k}}(f+\tilde{f})^{2}\right)^{1 / 2}\left(E_{V_{k}} \otimes \tilde{E}_{V_{k}}(f-\tilde{f})^{2}\right)^{1 / 2} \\
& \leqq 2\left(E_{V_{k}} f^{2}\right)^{1 / 2}\left(c_{0} \cdot E_{V_{k}}\left|B_{V_{k}} f\right|^{2}\right)^{1 / 2}
\end{aligned}
$$

where $c_{0} \equiv c_{0}\left(\left|V_{k}\right|\right)$. In the last step of (1.92) we used the fact that $\tilde{E}_{V_{k}}$ is an isomorphic copy of $E_{V_{k}}$ together with the triangle inequality and the mass gap inequality for the measure $E_{V_{k}} \otimes \widetilde{E}_{V_{k}}$ (see (1.52)). Using (1.91), (1.92) and (1.88) together with the fact that

$$
\left\|\bar{B}_{j} U_{V_{k}}-B_{j} \tilde{U}_{V_{k}}\right\|_{\infty} \leqq 2\|\Phi\|
$$

we obtain the bound

$$
|(1.75)| \leqq 4\|\Phi\| e^{4\|\Phi\|} c_{0}^{1 / 2} \gamma^{L}\left(E_{V_{k}} f^{2}\right)^{1 / 2}\left(E_{V_{k}}\left|B_{V_{k}} f\right|^{2}\right)^{1 / 2} .
$$

From this, (1.65), (1.64) and Hoelder inequality for the measure $E_{\tilde{\Gamma}^{(n)}}$ we get

$$
\left|B_{j} E_{\Gamma^{(n)}} f^{2}\right| \leqq 4\|\Phi\| e^{4\|\Phi\|} c_{0}^{1 / 2} \gamma^{L}\left(E_{\Gamma^{(n)}} f^{2}\right)^{1 / 2}\left(E_{\Gamma^{(n)}}\left|B_{V_{k}} f\right|^{2}\right)^{1 / 2}
$$

Since

$$
\left(E_{\Gamma^{(n)}} f^{2}\right)^{1 / 2} \leqq e^{\|\Phi\|} A_{j}\left(E_{\Gamma^{(n)}} f^{2}\right)^{1 / 2},
$$

so by the same arguments as in (1.31) and (1.32) we get

$$
\left|B_{j}\left(E_{\Gamma^{(n)}} f^{2}\right)^{1 / 2}\right| \leqq 2\|\Phi\| e^{5\|\Phi\|} c_{0}^{1 / 2} \gamma^{L}\left(E_{\Gamma^{(n)}}\left|V_{V_{k}} f\right|^{2}\right)^{1 / 2}
$$

Squaring (1.97) and summing over $j \in \Gamma^{(n+1)}$ taking into account (1.60) we obtain

$$
\left|B_{\Gamma^{(n+1)}}\left(E_{\Gamma^{(n)}} f^{2}\right)^{1 / 2}\right|^{2} \leqq \lambda E_{\Gamma^{(n)}}\left|B_{\Gamma^{(n)}} f\right|^{2}
$$

with

$$
\lambda \equiv 8 r\|\Phi\|^{2} e^{10\|\Phi\|} c_{0} \gamma^{2 L} .
$$

By Lemma 1.1 and the fact that

$$
\left|V_{k}\right| \leqq 4(L+1) r
$$


our $c_{0} \equiv c_{0}\left(\left|V_{k}\right|\right)$ satisfies

$$
0<c_{0}<C \log (4(L+1) r) \text {. }
$$

Using (1.101), (1.99) together with (1.90) we conclude that we can get

$$
0<\lambda<1
$$

by taking $L$ sufficiently big.

This ends the proof of Lemma 1.2b.

\section{Concluding Remarks}

We have shown that each Gibbs measure of an infinite discrete spin system with finite range interactions (at any temperature) on a one dimensional lattice satisfies the log-Sobolev inequality. The same result is expected to hold for continuous spins. (Some details of the proof are even simpler in this case.)

Let us stress that, despite the fact that we considered above a one dimensional system, the results of the present paper concern in a sense a more general situation than the one of [14], where the log-Sobolev inequalities have been proven for any lattice system in the Dobrushin uniqueness region. Namely in the situation considered in the present paper we work in Dobrushin-Shlosman uniqueness region. One may expect that the logarithmic Sobolev inequalities hold in Dobrushin-Shlosman uniqueness region for systems on an arbitrary lattice. This problem should be a subject of future investigations. It should be treated as a part of more general studies towards an understanding of the connections between dynamical and equilibrium description of statistical mechanical systems. In particular it would be interesting to show that there is a one to one correspondence between the structure of phases in the equilibrium description and structure of dynamical phases, the latter being distinguished (in the simplest case) by a rate of return to equilibrium in the corresponding dissipative dynamics. (For some issues connected to this programme as well as other interesting problems see also the discussion in [14].)

Acknowledgement. The author would like to thank Prof. S. Albeverio for his interest and critical reading of the manuscript.

\section{References}

1. Gross, L.: Logarithmic Sobolev inequalities. Am. J. Math. 97, 1061-1083 (1976)

2. Davies, E. B., Gross, L., Simon, B.: Hypercontractivity: A bibliographical review, To appear in Proceedings of the Hoegh-Krohn Memorial Conference

3. Nelson, E.: The free Markov field. J. Funct. Anal. 12, 211-227 (1973)

4. Simon, B., Hoegh-Krohn, R.: Hypercontractive semigroups and two dimensional self-coupled Bose fields. J. Funct. Anal. 9, 121-180 (1972)

5. Carlen, E. A., Stroock, D. W.: An application of the Barkry-Emery criterion to infinite dimensional diffusions, pp. 341-348 in Sem. de Probabilities XX. Azema J., Yor, M. (eds.) Lecture Notes in Mathematics, vol. 1204. Berlin, Heidelberg, New York: Springer

6. Bakry, E., Emery, M.: Hypercontractivite de semi-groupes des diffusion. C. R. Acad. Sci. Paris Ser. 1299, 775-777 (1984); Diffusions hypercontractives, pp. 177-206 in Sem. de Probabilities 
XIX. Azema, J., Yor, M. (eds.). Lecture Notes in Mathematics, vol. 1123. Berlin, Heidelberg, New York: Springer

7. Holley, R. A., Stroock, D. W.: $L_{2}$ Theory for stochastic Ising model. Z. Wahr. v. Geb. 35, 87-101 (1976)

8. Holley, R. A., Stroock, D. W.: Application of the stochastic Ising model to the Gibbs states. Commun. Math. Phys. 48, 249-265 (1976)

9. Holley, R. A., Stroock, D. W.: In one and two dimensions, every stationary measure for a stochastic Ising model is a Gibbs state. Commun. Math. Phys. 55, 37-45 (1977)

10. Holley, R. A., Stroock, D. W.: Diffusions on an infinite dimensional torus. J. Funct. Anal. 42, 29-63 (1981)

11. Holley, R. A., Stroock, D. W.: Logarithmic Sobolev inequalities and stochastic Ising models. J. Stat. Phys. 46, 1159-1194 (1987)

12. Holley, R. A., Stroock, D. W.: Uniform and $L_{2}$ convergence in one dimensional stochastic Ising models. Commun. Math. Phys. 123, 85-93 (1989)

13. Zegarlinski, B.: On log-Sobolev inequalities for infinite lattice systems. Lett. Math. Phys. 20 (1990)

14. Zegarlinski, B.: Dobrushin uniqueness theorem and log-Sobolev inequalities, Bochum November 1989

15. Föllmer, H.: Phase transition and Martin boundary. In: Sem. de Probabilities Strasbourg IX. Lecture Notes in Mathematics, vol. 465. Berlin, Heidelberg, New York: Springer

16. Preston, Ch.: Random fields. Lecture Notes in Mathematics. vol. 534. Berlin, Heidelberg, New York: Springer

17. Dobrushin, R. L.: Prescribing a system of random variables by conditional distributions. Theor. Prob. Appl. 15, 453-486 (1970); The problem of uniqueness of a Gibbs random field and the problem of phase transition. Funct. Anal. Appl. 2, 302-312 (1968)

18. Landford III O. E.: Entropy and equilibrium states in classical statistical mechanics. In: Statistical mechanics and mathematical problems. Lenard A. (ed.) pp. 1-113 LNPh 20

19. Föllmer, H.: A covariance estimate for Gibbs measures. J. Funct. Anal. 46, 387-395 (1982)

20. Ruelle, D.: Statistical Mechanics: Rigorous Results. New York-Amsterdam: W. A. Benjamin 1969

21. Glauber, R. J.: Time dependent statistics of the Ising model. J. Math. Phys. 4, 294-307 (1963)

22. Rothaus, O. S.: Logarithmic Sobolev inequalities and the spectrum of Sturm-Liouville Operators. J. Funct. Anal. 39, 42-56 (1980); Logarithmic Sobolev inequalities and the spectrum of Schrödinger Operators. J. Funct. Anal. 42, 110-120 (1981)

23. Simon, B.: A remark on Nelson's best hypercontractive estimates. Proc. AMS 55, 376-378 (1978)

Communicated by M. Aizenman 